\title{
INTEGRATED DENSITY OF STATES FOR RANDOM METRICS ON MANIFOLDS
}

\section{DANIEL LENZ, NORBERT PEYERIMHOFF AND IVAN VESELIĆ}

\section{Introduction}

The mathematically rigorous study of random Schrödinger operators commenced in the 1970s. The motivation was to understand the transport properties of random media. Since then a variety of results on the spectral, wave-spreading and conductance properties of random Schrödinger operators have been derived on mathematical grounds. We refer to the textbook accounts $[\mathbf{8}, \mathbf{1 7}, \mathbf{5}, \mathbf{2 7}, \mathbf{3 2}]$ and the references cited therein.

This paper carries over the fundamental properties of random Schrödinger operators to random Laplace-Beltrami operators, that is, Laplacians with random metrics. Namely, we

(A) discuss a framework for ergodic, random operators on covering manifolds with randomness entering both via potential and metrics,

(B) show measurability of the introduced operators, which implies, in particular, almost sure constancy of their spectral features,

(C) prove existence and the selfaveraging property of the integrated density of states together with a Pastur-Šbin type trace formula.

Thereby we extend and apply the earlier $[\mathbf{2 8}, \mathbf{2 1}]$. The main result of this paper is result (C) concerning the integrated density of states (IDS). It has been announced in $[\mathbf{2 2}]$. Physically, the integrated density of states measures the number of electron energy levels per unit volume up to a given energy value. It can be obtained by a macroscopic limit, where ergodicity of the family of operators yields the selfaveraging nature, that is, the non-randomness, of this quantity. It is sometimes called the spectral density function.

Let us put these results in perspective. Probably the most prominent success of the theory of random Schrödinger operators is the proof of localization. This phenomenon has been explained on physical grounds by Anderson [3], but only in the late 1970s were first rigorous results established; see the original papers $[\mathbf{1 5}$, $\mathbf{1 4}, \mathbf{2}]$ or $[\mathbf{3 2}]$ for a monograph exposition.

In [10] Davies studies among others the relation of heat kernels on a manifold associated to different metrics. In this context he raises the question of localization due to random metrics. This should be analogous to the phenomena occurring in quantum wave guides $[\mathbf{1 9}]$.

In comparison to localization the study of the integrated density of states undertaken in this paper is a physically more basic and technically less involved question. Still this quantity comprises many important spectral features of the random Schrödinger operator and its understanding can be seen as a first step

Received 17 September 2002.

2000 Mathematics Subjects Classification 35J10, 58J35, 82B44. 
towards the proof of localization. Namely, the multiscale proof of localization of Fröhlich and Spencer [14] derived for (specific models of) random Schrödinger operators in Euclidean space relies on the continuity and asymptotic properties of the integrated density of states. These were first studied by Wegner [36], and respectively by Lifshitz [24]. In a forthcoming paper [20], we derive results on the (dis)continuity of the IDS for periodic and random operators on manifolds.

Let us note that Euclidean random divergence type operators do not cover our models, due to the more general geometry and underlying group structure we consider.

The paper is organized as follows. In the next section we introduce our model and state the main results. In $\S 3$ we introduce quadratic forms and derive the measurability of the quantities we are considering, thereby giving a precise form to (B) above. Section 4 is devoted to general results on random operators which are proven in an abstract setting in $[\mathbf{2 1}]$. This presents our treatment of (A) above. A discussion of heat kernels on manifolds is given in $\S 5$, specializing to the principle of not feeling the boundary in $\S 6$. We derive uniform bounds for the kernels of the semigroups of a random family of Schrödinger operators acting on a manifold and including singular non-negative potentials. Using these results, we then prove our main result concerning $(\mathrm{C})$ in $\S 7$.

\section{Model and results}

In this section we state the main results about the existence and nonrandomness of the integrated density of states. Beforehand we explain the geometric setting we are working in and the properties of the random Schrödinger operator.

Consider a complete Riemannian manifold $X$ of dimension $n$ with metric $g_{0}$ and associated volume form $\mathrm{vol}_{0}$. Let $\Gamma$ be a discrete infinite subgroup of the isometries of $\left(X, g_{0}\right)$, acting cocompactly, freely and properly discontinuously on $X$. Consequently, $M=X / \Gamma$ is a compact Riemannian manifold. Furthermore, let $\left(\Omega, \mathcal{B}_{\Omega}, \mathbb{P}\right)$ be a probability space on which $\Gamma$ acts ergodically by measure-preserving transformations.

Definition 2.1. A family of Riemannian metrics $\left\{g_{\omega}\right\}_{\omega \in \Omega}$ on $X$ with corresponding volume forms $\operatorname{vol}_{\omega}$ is called a random metric on $\left(X, g_{0}\right)$ if the following properties are satisfied.

(M1) The map $\Omega \times T M \rightarrow \mathbb{R},(\omega, v) \mapsto g_{\omega}(v, v)$ is jointly measurable.

(M2) There is a $C_{g} \in(0, \infty)$ such that

$$
C_{g}^{-1} g_{0}(v, v) \leqslant g_{\omega}(v, v) \leqslant C_{g} g_{0}(v, v) \quad \text { for all } \omega \in \Omega \text { and } v \in T X .
$$

(M3) There is a $C_{\rho}>0$ such that

$$
\left|\nabla_{0} \rho_{\omega}(x)\right|_{0} \leqslant C_{\rho} \text { for all } \omega \in \Omega \text { and } x \in X,
$$

where $\nabla_{0}$ denotes the gradient with respect to $g_{0}, \rho_{\omega}$ is the unique smooth density satisfying $d \operatorname{vol}_{0}=\rho_{\omega} d \operatorname{vol}_{\omega}$, and $|v|_{0}^{2}=g_{0}(v, v)$.

(M4) There is a uniform lower bound $K \in \mathbb{R}$ for the Ricci curvatures of all Riemannian manifolds $\left(X, g_{\omega}\right)$. 
(M5) The metrics are compatible in the sense that the deck transformations

$$
\gamma:\left(X, g_{\omega}\right) \rightarrow\left(X, g_{\gamma \omega}\right), \quad \gamma: x \mapsto \gamma x
$$

are isometries.

The property (M5) implies that, in particular, the induced maps

$$
U_{(\omega, \gamma)}: L^{2}\left(X, \operatorname{vol}_{\gamma^{-1} \omega}\right) \rightarrow L^{2}\left(X, \operatorname{vol}_{\omega}\right), \quad\left(U_{(\omega, \gamma)} f\right)(x)=f\left(\gamma^{-1} x\right)
$$

are unitary operators.

Based on this geometric setting, we consider a family of Schrödinger operators. These operators are defined via quadratic forms, as explained in $\S 3$.

Definition 2.2. Let $\left\{g_{\omega}\right\}$ be a random metric on $\left(X, g_{0}\right)$. For each $\omega \in \Omega$ let $H_{\omega}=\Delta_{\omega}+V_{\omega}$ be a Schrödinger operator defined on the Hilbert space $L^{2}\left(X, \operatorname{vol}_{\omega}\right)$. The operator $\left\{H_{\omega}\right\}_{\omega \in \Omega}$ is called a random (Schrödinger) operator if it satisfies the equivariance condition

$$
H_{\omega}=U_{(\omega, \gamma)} H_{\gamma^{-1} \omega} U_{(\omega, \gamma)}^{*},
$$

for all $\gamma \in \Gamma$ and $\omega \in \Omega$, and if the potential $V: \Omega \times X \rightarrow \mathbb{R}$ is jointly measurable and non-negative and $V_{\omega}=V(\omega, \cdot) \in L_{\mathrm{loc}}^{1}(X)$, for all $\omega \in \Omega$.

For technical reasons we require that the $\sigma$-algebra $\mathcal{B}_{\Omega}$ is countably generated. This can always be established by changing to an equivalent version of the defining stochastic processes given by the random potential and the random metric. This has been done explicitly for the potential in Remark 2.8 of [21].

In $\S 3$ we extend the standard notion of measurability for a family of operators acting on a fixed Hilbert space [18] to operators acting on varying Hilbert spaces. This leads to the following fundamental result.

TheOREM 1. A random operator $\left\{H_{\omega}\right\}_{\omega \in \Omega}$ is a measurable family of operators.

From this theorem and the results of [21] we immediately obtain the following result. (Note that $\sigma_{\mathrm{pp}}$ denotes the closure of the set of eigenvalues.)

Theorem 2. There exist $\Omega^{\prime} \subset \Omega$ of full measure and $\Sigma, \Sigma \bullet \subset \mathbb{R}$, such that $\sigma\left(H_{\omega}\right)=\Sigma$ and $\sigma_{\bullet}\left(H_{\omega}\right)=\Sigma$. for all $\omega \in \Omega^{\prime}$ where $\bullet=$ disc, ess, ac, sc, pp. Moreover, $\Sigma_{\text {disc }}=\emptyset$.

The above two theorems and the framework underlying their proofs complete our investigation of (A) and (B) of the introduction.

Next, we introduce the (abstract) density of states for a random operator $\left\{H_{\omega}\right\}$ as the measure on $\mathbb{R}$, given by

$$
\rho_{H}(f):=\frac{\mathbb{E}\left[\operatorname{tr}\left(\chi_{\mathcal{F}} f\left(H_{\bullet}\right)\right)\right]}{\mathbb{E}\left[\operatorname{vol}_{\bullet}(\mathcal{F})\right]}, \quad \text { for } f \text { bounded, measurable. }
$$

Here $\mathcal{F} \subset X$ is a precompact $\Gamma$-fundamental domain with piecewise smooth boundary, $\mathbb{E}$ denotes the expectation with respect to $\mathbb{P}$ and $\operatorname{tr}=\operatorname{tr}_{\omega}$ is the trace on the Hilbert space $L^{2}\left(X, \operatorname{vol}_{\omega}\right)$, where we suppress the index $\omega$ in the following. The 
expression (2) is closely related to a trace $\tau$ of a von Neumann algebra, as discussed in $\S 4$ and summarized in the next theorem.

THEOREM 3. The function $\rho_{H}$ is a spectral measure for the direct integral operator

$$
H:=\int_{\Omega}^{\oplus} H_{\omega} d \mathbb{P}(\omega)
$$

and $\rho_{H}(f)$ and $\tau(f(H))$ coincide for arbitrary bounded measurable $f$ on $\mathbb{R}$, up to a fixed constant factor. In particular, the almost sure spectrum $\Sigma$ coincides with the topological support $\{\lambda \in \mathbb{R} \mid \rho((\lambda-\varepsilon, \lambda+\varepsilon))>0$ for all $\varepsilon>0\}$ of $\rho_{H}$.

Recall that a measure $\phi$ on $\mathbb{R}$ is a spectral measure for a selfadjoint operator $H$ with spectral family $E_{H}$ if, for Borel measurable $B \subset \mathbb{R}$,

$$
\phi(B)=0 \quad \Longleftrightarrow \quad E_{H}(B)=0 .
$$

To state our main result (see $(\mathrm{C})$ of the introduction), it is indispensable to assume that the underlying discrete group $\Gamma$ is amenable. We introduce restrictions of operators on $X$ to open sets $D \subset X$ with $\operatorname{vol}_{\omega}(D)<\infty$. The restriction of $H_{\omega}$ to $D$ with Dirichlet boundary conditions (b.c.) will be denoted by $H_{\omega}^{D}$. The restriction $H_{\omega}^{D}$ is again selfadjoint, bounded below and has purely discrete spectrum. Therefore, we may enumerate its eigenvalues in increasing order, counting multiplicities: $\lambda_{1}\left(H_{\omega}^{D}\right) \leqslant \lambda_{2}\left(H_{\omega}^{D}\right) \leqslant \ldots \leqslant \lambda_{i}\left(H_{\omega}^{D}\right) \rightarrow \infty$. We define the normalized eigenvalue counting function as

$$
N_{\omega}^{D}(\lambda)=\frac{\#\left\{i \mid \lambda_{i}\left(H_{\omega}^{D}\right)<\lambda\right\}}{\operatorname{vol}_{\omega}(D)} .
$$

Thus $N_{\omega}^{D}$ is a distribution function and has countably many discontinuity points.

Amenability of $\Gamma$ guarantees the existence of an exhaustion of $X$ by open sets $\left\{D^{j}\right\}_{j}$ with very strong additional properties; see $\S 7$. Such an exhaustion $\left\{D^{j}\right\}_{j}$ is called an admissible sequence of subsets of $X$. For the associated restricted operators we use the shorthand $H_{\omega}^{j}=H_{\omega}^{D^{j}}$ and, similarly, $N_{\omega}^{j}=N_{\omega}^{D^{j}}$. Our main result establishes the selfaveraging property of the IDS and expresses it by a Subin type trace formula $[\mathbf{3 3}, \mathbf{3 4}]$.

TheOREm 4. Let $\left\{D^{j}\right\}_{j}$ be an admissible sequence and $\left\{H_{\omega}\right\}_{\omega}$ be as above. There exists a set $\Omega^{\prime}$ of full measure such that

$$
\lim _{j \rightarrow \infty} N_{\omega}^{j}(\lambda)=\rho_{H}((-\infty, \lambda)),
$$

for every $\omega \in \Omega^{\prime}$ and every point $\lambda \in \mathbb{R}$ with $\rho_{H}(\{\lambda\})=0$.

The distribution function of $\rho_{H}$ is denoted by $N_{H}$ and is called the integrated density of states (IDS) of the random operator $\left\{H_{\omega}\right\}$, that is,

$$
N_{H}(\lambda)=\rho_{H}((-\infty, \lambda)) .
$$

Since $N_{H}$ can be obtained by an exhaustion procedure $D^{j} \rightarrow X$ without integrating over $\Omega$ explicitly, it is called selfaveraging. The proof of Theorem 4 in $\S 7$ actually also establishes the following result. For a set $D^{j} \subset X$ in an 
admissible sequence denote

$$
N_{\omega}^{j, f}(\lambda):=\frac{\operatorname{tr}\left(\chi_{D^{j}} E_{\omega}(\lambda)\right)}{\operatorname{vol}_{\omega}\left(D^{j}\right)}
$$

Here, the superscript $f$ stands for the fact that this finite volume IDS is defined without the use (that is, free) of boundary conditions, and $E_{\omega}$ denotes the spectral family of $H_{\omega}$.

Corollary 2.3. For almost every $\omega \in \Omega$ the convergence

$$
\lim _{j \rightarrow \infty} N_{\omega}^{j, f}(\lambda)=N_{H}(\lambda)
$$

holds at every continuity point $\lambda$ of $N_{H}$.

This means that in the macroscopic limit $D^{j} \rightarrow X$, it is not noticed whether the restriction of the operator in space, or the projection on an energy interval took place first.

For simplicity, we have so far assumed the potentials $V$ to be non-negative. It suffices to assume that the $V_{\omega}$ are uniformly bounded below by a constant $C$ not depending on $\omega \in \Omega$. Then our results apply to the shifted operator family $\left\{H_{\omega}-C\right\}_{\omega \in \Omega}$. Since $N_{H-C}(\lambda-C)=N_{H}(\lambda)$ for all $\lambda \in \mathbb{R}$, and similarly for the normalized eigenvalue counting functions, the results carry over to the original operators.

\section{Quadratic forms and measurability}

In this section we give a precise definition of the operators we are dealing with and show their measurability.

To introduce our operators, we will use quadratic forms. The relevant theory can be found, in, for example, the first two sections of [9]. It is developed there for $X=\mathbb{R}^{n}$ but carries over directly to arbitrary manifolds $X$.

We abbreviate the scalar product in the tangent space by

$$
\langle v, w\rangle_{\omega}:=g_{\omega}(x)(v, w) \text { for all } v, w \in T_{x} X .
$$

For $D \subset X$ open and each $\omega \in \Omega$ we define the quadratic forms

$$
\widetilde{Q}\left(\Delta_{\omega}^{D}\right): C_{c}^{\infty}(D) \times C_{c}^{\infty}(D) \rightarrow \mathbb{R}, \quad(f, h) \mapsto \int_{D}\langle\nabla f(x), \nabla h(x)\rangle_{\omega} d \operatorname{vol}_{\omega}(x)
$$

and

$$
\widetilde{Q}\left(V_{\omega}^{D}\right): C_{c}^{\infty}(D) \times C_{c}^{\infty}(D) \rightarrow \mathbb{R}, \quad(f, h) \mapsto \int_{D} f(x) V_{\omega}(x) h(x) d \operatorname{vol}_{\omega}(x) .
$$

These forms are closable and their closures $Q\left(\Delta_{\omega}^{D}\right)$ and $Q\left(V_{\omega}^{D}\right)$, respectively, give rise to selfadjoint non-negative operators $\Delta_{\omega}^{D}$ and $V_{\omega}$. Next, consider the form

$$
\widetilde{Q}\left(H_{\omega}^{D}\right): C_{c}^{\infty}(D) \times C_{c}^{\infty}(D) \rightarrow \mathbb{R}, \quad(f, h) \mapsto Q\left(\Delta_{\omega}^{D}\right)(f, h)+Q\left(V_{\omega}^{D}\right)(f, h) .
$$

This form is closable, the closure $Q\left(H_{\omega}^{D}\right)$ is the form sum of $Q\left(\Delta_{\omega}^{D}\right)$ and $Q\left(V_{\omega}^{D}\right)$, and $Q\left(H_{\omega}^{D}\right)$ induces, again, a selfadjoint operator (see [9, Theorem 1.8.1]). Of course, for smooth $V$ and $f \in C_{c}^{\infty}(D)$ we have $H_{\omega}^{D} f(x)=\Delta_{\omega} f(x)+V(x) f(x)$. The form $Q\left(H_{\omega}^{D}\right)$ is a Dirichlet form (see [9, Theorem 1.3.5]), that is, it satisfies

$$
\exp \left(-t H_{\omega}\right): L^{\infty}\left(D, \operatorname{vol}_{\omega}\right) \rightarrow L^{\infty}\left(D, \operatorname{vol}_{\omega}\right) \text { is a contraction for every } t>0
$$


and

$\exp \left(-t H_{\omega}\right): L^{2}\left(D, \operatorname{vol}_{\omega}\right) \rightarrow L^{2}\left(D, \operatorname{vol}_{\omega}\right)$ is positivity preserving for every $t>0$.

Semigroups $e^{-t H}$ associated to Dirichlet forms are called symmetric Markov semigroups.

There exist positive, smooth functions $\rho_{\omega} \in C^{\infty}(X)$ such that

$$
\int_{X} f(x) d \operatorname{vol}_{0}(x)=\int_{X} f(x) \rho_{\omega}(x) d \operatorname{vol}_{\omega}(x) .
$$

More explicitly, $\rho_{\omega}(x)$ is given by

$$
\rho_{\omega}(x)=\left(\operatorname{det} g_{0}\left(e_{i}^{\omega}, e_{j}^{\omega}\right)\right)^{1 / 2}=\left(\operatorname{det} g_{\omega}\left(e_{i}^{0}, e_{j}^{0}\right)\right)^{-1 / 2},
$$

where $e_{1}^{0}, \ldots, e_{d}^{0} \in T_{x} X$ is any base of $T_{x} X$ orthonormal with respect to $g_{0}$ and $e_{1}^{\omega}, \ldots, e_{d}^{\omega} \in T_{x} X$ is any base orthonormal with respect to $g_{\omega}$. Consequently, the operators

$$
S_{\omega}: L^{2}\left(D, \operatorname{vol}_{0}\right) \rightarrow L^{2}\left(D, \operatorname{vol}_{\omega}\right), \quad S_{\omega}(f)=\rho_{\omega}^{1 / 2} f
$$

are unitary. The $L^{2}$-products on $L^{2}\left(D, \mathrm{vol}_{0}\right)$ and on $L^{2}\left(D, \operatorname{vol}_{\omega}\right)$ are denoted by $(\cdot, \cdot)_{0}$ and $(\cdot, \cdot)_{\omega}$, respectively. The corresponding norms are denoted by $\|\cdot\|_{0}$ and $\|\cdot\|_{\omega}$.

It follows from property (M2) of Definition 2.1 that

$$
C_{g}^{-n / 2} \leqslant \rho_{\omega}(x) \leqslant C_{g}^{n / 2} \text { for all } x \in D, \omega \in \Omega .
$$

Now we introduce the notion of measurability of a family of selfadjoint operators, indexed by the elements of $\Omega$. It is a modification of the definition from [18] to operators with varying domains of definition.

Definition 3.1. A family of selfadjoint operators $\left\{H_{\omega}\right\}_{\omega}$, where the domain of $H_{\omega}$ is a dense subspace $\mathcal{D}_{\omega}$ of $L^{2}\left(D, \operatorname{vol}_{\omega}\right)$, is called a measurable family of operators if

$$
\omega \mapsto\left(f_{\omega}, F\left(H_{\omega}\right) f_{\omega}\right)_{\omega}
$$

is measurable for all bounded, measurable functions $F: \mathbb{R} \rightarrow \mathbb{C}$ and all $f: \Omega \times D \rightarrow \mathbb{R}$ measurable with $f_{\omega} \in L^{2}\left(D, \operatorname{vol}_{\omega}\right)$ and $f_{\omega}(x)=f(\omega, x)$, for every $\omega \in \Omega$.

REMARK 3.2. In our setting, due to (M2), the above definition can be slightly simplified. Namely, a family of operators $\left\{H_{\omega}\right\}_{\omega}$ is measurable if and only if

$$
\omega \mapsto\left(f, F\left(H_{\omega}\right) f\right)_{\omega} \text { is measurable }
$$

for all $F: \mathbb{R} \rightarrow \mathbb{C}$ with $F \in L^{\infty}$ and all $f \in L^{2}\left(D, \operatorname{vol}_{0}\right)$. (Note that, due to (M2), $L^{2}\left(D, \operatorname{vol}_{0}\right)$ and $L^{2}\left(D, \operatorname{vol}_{\omega}\right)$ coincide for all $\omega \in \Omega$ as sets, though not in their scalar product.)

To see this, note that (7) implies the same statement for $f(\omega, x)$ replaced by $h(\omega, x)=g(\omega) f(x)$ where $g \in L^{2}(\Omega)$ and $f \in L^{2}\left(D, \operatorname{vol}_{0}\right)$. Such functions form a total set in $L^{2}(\Omega \times D, \mathbb{P} \circ \mathrm{vol})$.

Now, consider a measurable $h: \Omega \times D \rightarrow \mathbb{R}$ such that $h_{\omega}:=h(\omega, \cdot) \in L^{2}\left(D, \operatorname{vol}_{\omega}\right)$ for every $\omega \in \Omega$. Then $h^{n}(\omega, x):=\chi_{h, n}(\omega) h(\omega, x)$ is in $L^{2}(\Omega \times D, \mathbb{P} \circ$ vol $)$ where $\chi_{h, n}$ denotes the characteristic function of the set $\left\{\omega \mid\left\|h_{\omega}\right\|_{L^{2}\left(D, \mathrm{vol}_{\omega}\right)} \leqslant n\right\}$. Since $\chi_{h, n} \rightarrow 1$ 
pointwise on $\Omega$ for $n \rightarrow \infty$, we obtain

$$
\left(h_{\omega}^{n}, F\left(H_{\omega}\right) h_{\omega}^{n}\right)_{\omega} \rightarrow\left(h_{\omega}, F\left(H_{\omega}\right) h_{\omega}\right)_{\omega}
$$

which shows that $\left\{H_{\omega}\right\}_{\omega}$ is a measurable family of operators.

The following proposition (and its proof) is a variant of Proposition 3 in [18]. It suits our purposes and shows that our notion of measurability is compatible with that of Kirsch and Martinelli. Let $\left\{A_{\omega}\right\}_{\omega}$ be a family of densely defined non-negative selfadjoint operators on a fixed Hilbert space $\mathcal{H}$. Denote by $\widetilde{\Sigma}=\overline{\bigcup_{\omega} \sigma\left(A_{\omega}\right)}$ the closure of all spectra and by $\mathcal{F}_{i}$ the following classes of functions:

$$
\begin{aligned}
& \mathcal{F}_{1}=\left\{\chi_{(-\infty, \lambda)} \mid \lambda \geqslant 0\right\}, \\
& \mathcal{F}_{2}=\left\{x \mapsto e^{i t x} \mid t \in \mathbb{R}\right\}, \\
& \mathcal{F}_{3}=\left\{x \mapsto e^{-t x} \mid t \geqslant 0\right\}, \\
& \mathcal{F}_{4}=\left\{x \mapsto(z-x)^{-1} \mid z \in \mathbb{C} \backslash \widetilde{\Sigma}\right\}, \\
& \mathcal{F}_{5}=\mathcal{F}_{4}\left(z_{0}\right)=\left\{x \mapsto\left(z_{0}-x\right)^{-1}\right\} \text { for a fixed } z_{0} \in \mathbb{C} \backslash \widetilde{\Sigma}, \\
& \mathcal{F}_{6}=C_{b}=\{f: \mathbb{R} \rightarrow \mathbb{C} \mid f \text { bounded, continuous }\}, \\
& \mathcal{F}_{7}=L^{\infty}=\{f: \mathbb{R} \rightarrow \mathbb{C} \mid f \text { bounded, measurable }\} .
\end{aligned}
$$

Proposition 3.3. The following properties are equivalent:

$$
\omega \mapsto\left\langle f, F\left(A_{\omega}\right) h\right\rangle_{\mathcal{H}} \text { is measurable for all } f, h \in \mathcal{H} \text { and } F \in \mathcal{F}_{i},
$$

where $i=1, \ldots, 7$.

Proof. For the equivalence of $\left(\mathbf{F}_{4}\right)$ and $\left(\mathbf{F}_{5}\right)$ we assume $d\left(z_{0}, \widetilde{\Sigma}\right)=\delta$ and that $\left(z_{0}-H_{\omega}\right)^{-1}$ is weakly measurable. Using a Neumann series expansion as in $[\mathbf{3 0}$, Theorem VI.5] one infers the weak measurability of $\left(z-H_{\omega}\right)^{-1}$ for all $z$ with $d\left(z, z_{0}\right)<\delta$. Iterating this argument, we obtain measurability of $\left(z-H_{\omega}\right)^{-1}$ for all $z \in \mathbb{C} \backslash \widetilde{\Sigma}$.

Now, by the Stone-Weierstrass theorem we obtain the equivalence of $\left(\mathbf{F}_{2}\right)$, $\left(\mathbf{F}_{3}\right),\left(\mathbf{F}_{4}\right),\left(\mathbf{F}_{5}\right),\left(\mathbf{F}_{6}\right)$.

The equivalence of $\left(\mathbf{F}_{1}\right)$ and $\left(\mathbf{F}_{7}\right)$ follows by monotone class arguments.

As $\left(\mathbf{F}_{7}\right) \Rightarrow\left(\mathbf{F}_{6}\right)$ is clear, it only remains to prove $\left(\mathbf{F}_{6}\right) \Rightarrow\left(\mathbf{F}_{1}\right)$. This is immediate as every characteristic function $\chi_{(-\infty, \lambda)}$ is a pointwise monotone limit of continuous functions.

We prove now that the random operator $\left\{H_{\omega}\right\}$ introduced in $\S 2$ is measurable in the sense of Definition 3.1. The first step in the proof is to pull all operators $\left\{H_{\omega}\right\}_{\omega}$ on the same Hilbert space by the unitary transformation $S_{\omega}$ and to show the following comparability property of the associated quadratic forms.

Proposition 3.4. Let the selfadjoint operators

$$
A_{\omega}:\left(S_{\omega}\right)^{-1} \mathcal{D}\left(\Delta_{\omega}^{D}\right) \subset L^{2}\left(D, \operatorname{vol}_{0}\right) \rightarrow L^{2}\left(D, \operatorname{vol}_{0}\right)
$$

be defined by $A_{\omega}:=\left(S_{\omega}\right)^{-1} \Delta_{\omega}^{D} S_{\omega}$. Let $Q_{0}$ and $Q_{\omega}$ be the quadratic forms 
associated to the operators $\Delta_{0}^{D}$ and $A_{\omega}$. Then there is a constant $C_{A}$ such that

$$
C_{A}^{-1}\left(Q_{0}(f, f)+\|f\|_{0}^{2}\right) \leqslant Q_{\omega}(f, f)+\|f\|_{0}^{2} \leqslant C_{A}\left(Q_{0}(f, f)+\|f\|_{0}^{2}\right),
$$

for all $f \in C_{c}^{\infty}(D)$ and $\omega \in \Omega$. Moreover, there exists a dense subspace $\mathcal{D} \subset L^{2}\left(D, \operatorname{vol}_{0}\right)$ with $\mathcal{D}=\mathcal{D}\left(A_{\omega}^{1 / 2}\right)=\mathcal{D}\left(\left(\Delta_{0}^{D}\right)^{1 / 2}\right)$ for every $\omega \in \Omega$ and (8) holds for every $f \in \mathcal{D}$.

Proof. Direct calculation for $f \in C_{c}^{\infty}(D)$ shows that

$$
Q_{\omega}(f, f)=\left(S_{\omega} f, \Delta_{\omega} S_{\omega} f\right)_{\omega} \leqslant 2\left(\left\|\rho_{\omega}^{1 / 2} \nabla_{\omega} f\right\|_{\omega}^{2}+\left\|f \nabla_{\omega} \rho_{\omega}^{1 / 2}\right\|_{\omega}^{2}\right) .
$$

To bound $\left\|\rho_{\omega}^{1 / 2} \nabla_{\omega} f\right\|_{\omega}^{2}$ we consider the $n \times n$-matrix $A=\left(a_{i j}\right)$ defined by $e_{i}^{0}=\sum_{j=1}^{n} a_{i j} e_{j}^{\omega}$. A calculation using (M2) in Definition 2.1 shows that $C_{g}^{-1} \leqslant A A^{\top} \leqslant C_{g}$. This implies that

$$
C_{g}^{-1}\left|\nabla_{\omega} f(x)\right|_{\omega}^{2} \leqslant\left|\nabla_{0} f(x)\right|_{0}^{2} \leqslant C_{g}\left|\nabla_{\omega} f(x)\right|_{\omega}^{2},
$$

and thus

$$
\begin{aligned}
\left\|\rho_{\omega}^{1 / 2} \nabla_{\omega} f\right\|_{\omega}^{2} & =\int_{D}\left|\nabla_{\omega} f(x)\right|_{\omega}^{2} d \operatorname{vol}_{0}(x) \\
& \leqslant C_{g} \int_{D}\left|\nabla_{0} f(x)\right|_{0}^{2} d \operatorname{vol}_{0}(x)=C_{g} Q_{0}(f, f) .
\end{aligned}
$$

To estimate $\left\|f \nabla_{\omega} \rho_{\omega}^{1 / 2}\right\|_{\omega}^{2}$ we use (5), (10) and (M3) of Definition 2.1 to calculate

$$
\left\|f \nabla_{\omega} \rho_{\omega}^{1 / 2}\right\|_{\omega}^{2} \leqslant C_{g}^{1+n / 2} \int_{D}\left|\nabla_{0} \rho_{\omega}(x)\right|_{0}^{2} f^{2}(x) d \operatorname{vol}_{\omega}(x) \leqslant C_{g}^{1+n} C_{\rho}^{2}\|f\|_{0}^{2} .
$$

By symmetry, there is also an estimate of the form

$$
C_{A}^{-1}\left(Q_{0}(f, f)+\|f\|_{0}^{2}\right) \leqslant Q_{\omega}(f, f)+\|f\|_{\omega}^{2},
$$

for all $f \in C_{c}^{\infty}(D)$, and the first statement is proven. The statement follows now from (8), as $C_{c}^{\infty}(D)$ is a core for $Q_{0}$ and $Q_{\omega}$.

Proposition 3.5 (see [32, Proposition 1.2.6]). Let $Q_{\omega}$, with $\omega \in \Omega$, and $Q_{0}$ be non-negative closed quadratic forms with the following properties:

(P1) $Q_{\omega}$, with $\omega \in \Omega$, and $Q_{0}$ are defined on the same dense subset $\mathcal{D}$ of a fixed Hilbert space $\mathcal{H}$;

(P2) there is a fixed constant $C>0$ such that

$$
C^{-1}\left(Q_{0}(f, f)+\|f\|_{0}^{2}\right) \leqslant Q_{\omega}(f, f)+\|f\|_{0}^{2} \leqslant C\left(Q_{0}(f, f)+\|f\|_{0}^{2}\right) ;
$$

(P3) the map $\omega \mapsto Q_{\omega}(f, f)$ is measurable, for every $f \in \mathcal{D}$.

Then the family $\left\{H_{\omega}\right\}_{\omega}$ of associated selfadjoint operators satisfies the equivalent properties of Proposition 3.3.

The foregoing propositions allow us to show the following.

Proposition 3.6. The family $\left\{A_{\omega}\right\}_{\omega}$ of Proposition 3.4 is a measurable family of operators.

Proof. Since $C_{c}^{\infty}(D)$ is a core for $Q_{\omega}$ for all $\omega$, the closures of this set with respect to one of the equivalent norms in (8) coincide, which shows assumption 
(P1) of Proposition 3.5. Property (P2) is just (8) and (P3) is obvious for $f \in C_{c}^{\infty}(D)$. The required result then follows by approximation for all $f \in \mathcal{D}$.

Proof of Theorem 1. For $n \in \mathbb{N}$ and $\omega \in \Omega$, define bounded functions $V_{\omega}^{n}: X \rightarrow \mathbb{R}$ by $V_{\omega}^{n}(x):=\min \left\{n, V_{\omega}(x)\right\}$. Thus, the operator sum $A_{\omega}^{n}:=A_{\omega}+V_{\omega}^{n}$ is well defined, where $A_{\omega}$ is as in Proposition 3.4 and $D=X$. Moreover, by [18, Proposition 2.4] and Proposition 3.6, the family of operators $A_{\omega}^{n}$ is measurable. In particular, the corresponding semigroups $\omega \mapsto \exp \left(-t A_{\omega}^{n}\right)$, for $t>0$, are weakly measurable. Now, obviously, the forms of $A_{\omega}^{n}$ converge monotonically towards the form of $A_{\omega}^{\infty}:=A_{\omega}+V_{\omega}$. By [16, Theorems VIII.3.13a and IX.2.16], this implies that the semigroups of $A_{\omega}^{n}$ converge weakly towards the semigroup $\omega \mapsto \exp \left(-t A_{\omega}^{\infty}\right)$ for $n \rightarrow \infty$, and the measurability of the family $A_{\omega}^{\infty}$ follows. Finally, this implies measurability of the family $H_{\omega}$, since $H_{\omega}=S_{\omega} A_{\omega}^{\infty} S_{\omega}{ }^{-1}$ and $S_{\omega}$ is multiplication with the measurable function $(x, \omega) \mapsto \rho_{\omega}(x)^{1 / 2}$.

The same arguments show measurability of the restricted operators $\left\{H_{\omega}^{D}\right\}_{\omega}$.

\section{Abstract spectral properties of random operators}

We saw in the last section that a random operator $\left\{H_{\omega}\right\}_{\omega}$ is a measurable family of operators. This enables us to make use of the results derived in [21] for random operators in an abstract setting. The following information can be inferred from the cited source.

Definition 4.1. A family $\left\{A_{\omega}\right\}_{\omega \in \Omega}$ of bounded operators

$$
A_{\omega}: L^{2}\left(X, \operatorname{vol}_{\omega}\right) \rightarrow L^{2}\left(X, \operatorname{vol}_{\omega}\right)
$$

is called a bounded random operator if it satisfies:

(i) $\omega \mapsto\left\langle g_{\omega}, A_{\omega} f_{\omega}\right\rangle$ is measurable for arbitrary $f, g \in L^{2}(\Omega \times X, \mathbb{P} \circ$ vol $)$;

(ii) there exists a $C \geqslant 0$ with $\left\|A_{\omega}\right\| \leqslant C$ for almost all $\omega \in \Omega$;

(iii) for all $\omega \in \Omega$ and $\gamma \in \Gamma$ the equivariance condition $A_{\omega}=U_{(\omega, \gamma)} A_{\gamma^{-1} \omega} U_{(\omega, \gamma)}^{*}$ is satisfied.

Two bounded random operators $\left\{A_{\omega}\right\}_{\omega}$ and $\left\{B_{\omega}\right\}_{\omega}$ are called equivalent, $\left\{A_{\omega}\right\}_{\omega} \sim\left\{B_{\omega}\right\}_{\omega}$, if $A_{\omega}=B_{\omega}$ for $\mathbb{P}$-almost every $\omega \in \Omega$. Each equivalence class of bounded random operators $\left\{A_{\omega}\right\}_{\omega}$ gives rise to a bounded operator $A$ on $L^{2}(\Omega \times X, \mathbb{P} \circ \mathrm{vol})$ by $(A f)(\omega, x):=A_{\omega} f_{\omega}(x)$; see Appendix $\mathrm{A}$ in $[\mathbf{2 1}]$. This allows us to identify the equivalence class of $\left\{A_{\omega}\right\}_{\omega}$ with the bounded operator $A$.

By (1) and the last section, the resolvents, spectral projections and the semigroup associated to $\left\{H_{\omega}\right\}_{\omega}$ are all bounded random operators. Theorem 3.1 in [21] states that the set of bounded random operators forms a von Neumann algebra $\mathcal{N}$. Choose a measurable $u: \Omega \times X \rightarrow \mathbb{R}^{+}$with $\sum_{\gamma \in \Gamma} u_{\gamma^{-1} \omega}\left(\gamma^{-1} x\right) \equiv 1$ on $\Omega \times X$ and define the mapping

$$
\tau(A):=\mathbb{E}\left[\operatorname{tr}\left(u_{\bullet} A_{\bullet}\right)\right]
$$

on the set of non-negative operators in $\mathcal{N}$. This $\tau$ is independent of $u$ (chosen as above) and defines a trace on $\mathcal{N}$ of type $\mathrm{II}_{\infty}$, which is closely related to the IDS. Namely, the spectral projections $\left\{E_{\omega}(\lambda)\right\}_{\omega}$ onto the interval $(-\infty, \lambda)$ of a random operator $\left\{H_{\omega}\right\}_{\omega}$ form a bounded random operator. Thus it is an element of $\mathcal{N}$ and 
agrees with the spectral projection of $H:=\int_{\Omega}^{\oplus} H_{\omega} d \mathbb{P}(\omega)$ onto $(-\infty, \lambda)$. Hence $\tau(E(\lambda))$ is well defined and the choice $u_{\omega}(x)=\chi_{\mathcal{F}}(x)$ yields the identity $\tau(E(\lambda))=\mathbb{E}\left(\operatorname{vol}_{\bullet} \mathcal{F}\right) N_{H}(\lambda)$, where $\mathcal{F}$ is a fundamental domain as discussed after $(2)$.

Now, Theorems 2 and 3 follow from $\S \S 4$ and 5 of [21].

\section{Heat kernels}

In this section we investigate existence and properties of the kernels of the semigroups $\exp \left(-t H_{\omega}\right)$ and $\exp \left(-t H_{\omega}^{D}\right)$. It will be of particular importance to us to keep track of the dependence of the estimates both on the potential and the metric, since they vary with the random parameter $\omega \in \Omega$.

We start with the heat kernels of the Laplacians $\Delta_{\omega}$. Sobolev embedding theorems and spectral calculus directly show that

$$
\exp \left(-t \Delta_{\omega}\right): L^{2}\left(X, \operatorname{vol}_{\omega}\right) \rightarrow L^{\infty}\left(X, \operatorname{vol}_{\omega}\right) \text { is bounded for every } t>0 .
$$

Thus, $\exp \left(-t \Delta_{\omega}\right)$ is ultracontractive, and, by [9, Lemma 2.1.2], this implies that $\exp \left(-t \Delta_{\omega}\right)$ has a kernel $k_{\Delta_{\omega}}$ with

$$
0 \leqslant k_{\Delta_{\omega}}(t, x, y) \leqslant\left\|\exp \left(-t \Delta_{\omega}\right)\right\|_{1 \rightarrow \infty}=: C_{t}^{\omega}, \quad \text { for almost all } x, y \in X,
$$

where $\|A\|_{1, \infty}$ denotes the norm of $A: L^{1} \rightarrow L^{\infty}$. By the Trotter product formula we see that, for $f \geqslant 0, f \in L^{1}\left(X, \operatorname{vol}_{\omega}\right)$,

$$
0 \leqslant \exp \left(-t H_{\omega}\right) f(x) \leqslant \exp \left(-t \Delta_{\omega}\right) f(x) \leqslant C_{t}^{\omega}\|f\|_{L^{1}}
$$

for almost every $x \in X$. Thus, $\exp \left(-t H_{\omega}\right): L^{1}\left(X, \operatorname{vol}_{\omega}\right) \rightarrow L^{\infty}\left(X, \operatorname{vol}_{\omega}\right)$ is also bounded by $C_{t}^{\omega}$ and we have

$$
0 \leqslant k_{\omega}(t, x, y) \leqslant C_{t}^{\omega} \quad \text { for almost every } x, y \in X .
$$

To obtain a better estimate, we show that the $L^{2}$-kernel of the heat semigroup coincides with the fundamental solution of the heat equation as defined, in, for example, $[\mathbf{1 2}]$ or $[\mathbf{6}]$ in the case of the pure Laplacian. This allows us to apply estimates of $[\mathbf{2 3}]$ for the fundamental solution. Uniqueness of the fundamental solution and its agreement with the $L^{2}$-kernel are well known (see, for example, [12]). For completeness reasons, we give a short alternative functional analytic proof of this agreement based on a theorem of $[\mathbf{9}]$ in the more general case with a smooth potential $W$.

TheOREM 5. Let $\left\{H_{\omega}\right\}_{\omega}=\left\{\Delta_{\omega}+W_{\omega}\right\}_{\omega}$ be a random operator with smooth potential $W_{\omega} \in C^{\infty}(X)$. Then, the kernel $k_{\omega}$ has a non-negative representative in $C^{\infty}((0, \infty) \times X \times X)$. Moreover, we have:

(HE) $k_{\omega}$ is a solution of the heat equation

$$
\left(\frac{d}{d t}+\Delta_{\omega}^{y}+W_{\omega}\right) k_{\omega}(t, x, y)=0,
$$

where $\Delta_{\omega}^{y}$ denotes the $\Delta_{\omega}$ operator acting on the variable $y$;

(W) $k_{\omega}(t, x, \cdot)$ converges weakly to the point mass in $x$ :

$$
\int k_{\omega}(t, x, y) f(y) d y \rightarrow f(x), \quad \text { as } t \rightarrow 0,
$$

for every bounded continuous $f$ on $X$ and every $x \in X$. 
Proof. Mimicking the proof of [9, Theorem 5.2.1], we infer that $k_{\omega}$ has a representative in $C^{\infty}((0, \infty) \times X \times X)$. Since $\exp \left(-t H_{\omega}\right)$ is positivity preserving, we conclude that $k_{\omega} \geqslant 0$. Now, direct calculations show (HE). To show (W), we recall that $\exp \left(-t H_{\omega}\right)$ is a contraction. Thus, by standard measure theory (see, for example, [4, Satz 30.8]) it suffices to consider only $f \in C_{c}(X)$. We can restrict the set of functions even further to $f \in C_{c}^{\infty}(X)$, since $C_{c}^{\infty}(X)$ is dense in $C_{c}(X)$ with respect to the sup-norm. By elliptic regularity and Sobolev embeddings, there exist $a, b>0$ and $j \in \mathbb{N}$ with

$$
\left\|\exp \left(-t H_{\omega}\right) f-f\right\|_{\infty} \leqslant a\left\|H_{\omega}^{j}\left(\exp \left(-t H_{\omega}\right) f-f\right)\right\|_{2}+b\left\|\exp \left(-t H_{\omega}\right) f-f\right\|_{2}
$$

for every $t \geqslant 0$. By spectral calculus, the right-hand side tends to zero as $t \rightarrow 0$, and the theorem is proven.

To formulate the results of $\mathrm{Li}$ and Yau $[\mathbf{2 3}]$ that we will be using we denote by $d_{\omega}: X \times X \rightarrow[0, \infty)$ the Riemannian distance function on $X$ with respect to $g_{\omega}$, and similarly by $d_{0}$ the one with respect to the metric $g_{0}$.

Proposition 5.1. For every $t>0$ there exist constants $C_{t}>0$ and $\alpha_{t}>0$ with

$$
k_{\Delta_{\omega}}(t, x, y) \leqslant C_{t} \exp \left(-\alpha_{t} d_{\omega}^{2}(x, y)\right)
$$

for all $\omega \in \Omega$. In particular, the following hold:

(i) $C_{t}^{\omega} \leqslant C_{t}$ for every $\omega \in \Omega$, where $C_{t}^{\omega}$ was defined in (11);

(ii) for all $a>0$, there exists a $B_{t, a}<\infty$ such that the estimate

$$
\int_{X}\left[k_{\Delta_{\omega}}(t, x, y)\right]^{a} d \operatorname{vol}_{\omega}(y) \leqslant B_{t, a}
$$

holds uniformly in $x \in X$ and $\omega \in \Omega$; we set $B_{t}:=B_{t, 1}$.

Proof. Using [23, Corollary 3.1], property (M2) of Definition 2.1 and (5), we obtain the estimate (13) for the fundamental solution of the heat equation without potential. Note that the uniform lower bound $K>-\infty$ for the Ricci curvatures of $\left(X, g_{\omega}\right)$ enters into the constant $C_{t}$. By Theorem 5 the fundamental solution agrees with the $L^{2}$-kernel of the semigroup $\exp \left(-t \Delta_{\omega}\right)$. Given this estimate, (i) and (ii) are easy consequences. Note for (ii) that the volume of metric balls of radius $r$ can be estimated (uniformly in $\omega$ ) from above by $C_{1} \exp \left(C_{2} r\right)$ with fixed constants $C_{1}, C_{2}>0$, by property (M2), (5), and the Bishop volume comparison theorem (cf. [7, Theorem 3.9]).

Proposition 5.1 can be extended to the perturbed operator. To do so we will need the Feynman-Kac formula:

$$
e^{-t H_{\omega}} f(x)=\mathbf{E}_{x}\left(\exp \left(-\int_{0}^{t} V_{\omega}\left(X_{s}\right) d s\right) f\left(X_{t}\right)\right),
$$

where $\mathbf{E}_{x}$ denotes the expectation with respect to the Brownian motion $X_{t}$ starting in $x$. This formula on stochastically complete manifolds is presented in, for example, [13, Theorem IX.7A] for bounded continuous potentials. Using semigroup and integral convergence theorems in the same spirit as in the proof of 
[29, Theorem X.68], we can extend the validity of this formula to non-negative, locally $L^{1}$ potentials.

Corollary 5.2. For arbitrary open $D$ and $t>0$ the following holds:

$$
0 \leqslant k_{\omega}^{D}(t, x, y) \leqslant k_{\omega}(t, x, y) \leqslant C_{t} \exp \left(-\alpha_{t} d_{\omega}^{2}(x, y)\right),
$$

for almost all $x, y \in D$. The constants are as in the previous proposition. In particular, the integral estimate in Proposition 5.1(ii) also holds for the perturbed operator, for almost all $x \in X$.

Proof. Using the Feynman-Kac formula, we obtain

$$
\begin{aligned}
0 \leqslant \int_{X} k_{\omega}(t, x, y) f(y) d y & =\mathbf{E}_{x}\left(\exp \left(-\int_{0}^{t} V_{\omega}\left(X_{s}\right) d s\right) f\left(X_{t}\right)\right) \\
& \leqslant \mathbf{E}_{x}\left(f\left(X_{t}\right)\right)=\int_{X} k_{\Delta_{\omega}}(t, x, y) f(y) d y
\end{aligned}
$$

for all non-negative $f \in L^{2}\left(X, \operatorname{vol}_{\omega}\right)$. Proposition 5.1 implies that

$$
0 \leqslant k_{\omega}(t, x, y) \leqslant k_{\Delta_{\omega}}(t, x, y) \leqslant C_{t} \exp \left(-\alpha_{t} d_{\omega}^{2}(x, y)\right),
$$

for almost all $x, y \in X$. The inequality for the Dirichlet operator follows by socalled domain monotonicity; see for example [9, Theorem 2.1.6]. The proof of this theorem carries over directly from $\mathbb{R}^{n}$ to manifolds.

Finally, note that, for $\operatorname{vol}_{\omega}(D)<\infty$, the estimate $0 \leqslant k_{\omega}^{D}(t, x, y) \leqslant C_{t}$ implies that $\exp \left(-t H_{\omega}^{D}\right)$ is a Hilbert-Schmidt operator and thus $H_{\omega}^{D}$ has purely discrete spectrum by the spectral mapping theorem.

\section{The principle of not feeling the boundary}

In this section we show that the semigroups associated to our random operators satisfy a principle of not feeling the boundary.

Let $D$ be an open set on the manifold $X$. One expects the difference between the Dirichlet heat kernel $k_{H^{D}}(t, x, y)$ and $k_{H}(t, x, y)$ to be small as long as $t>0$ is small and $x$ and $y$ stay away from the boundary of $D$. This phenomenon is called the principle of not feeling the boundary. To treat it rigorously, we introduce the notion of thickened boundary. For $h>0$, let $\partial_{h} D:=\left\{x \in X \mid d_{0}(x, \partial D) \leqslant h\right\}$ and $D_{h}$ be the interior of the set $D \backslash \partial_{h} D$. Note that (M2) of Definition 2.1 implies the following inequalities:

$$
C_{g}^{-1} d_{0}(x, y) \leqslant d_{\omega}(x, y) \leqslant C_{g} d_{0}(x, y) .
$$

The main result of this section is the following.

TheOREM 6. For all $t, \varepsilon>0$, there exists an $h=h(t, \varepsilon)>0$ such that for every open set $D \subset X$ and all $\omega \in \Omega$, we have

$$
0 \leqslant k_{\omega}(t, x, y)-k_{\omega}^{D}(t, x, y) \leqslant \varepsilon,
$$

for almost all $x, y \in D_{h}$. 
The proof of Theorem 6 follows from the next two propositions. More precisely, in view of the next proposition, it is enough to prove the theorem for vanishing potential. This, however, is accomplished in Proposition 6.3.

Let $\tau_{x}^{D}$ denote the first exit time from $D$ for Brownian motion starting in $x$.

Proposition 6.1. The following statements are equivalent.

(i) For all $t, \varepsilon>0$, there exists an $h=h(t, \varepsilon)>0$ such that for every open set $D \subset X$ and all $\omega \in \Omega$, we have

$$
0 \leqslant k_{\Delta_{\omega}}(t, x, y)-k_{\Delta_{\omega}^{D}}(t, x, y) \leqslant \varepsilon,
$$

for almost all $x, y \in D_{h}$.

(ii) For all $t, \varepsilon>0$, there exists an $h=h(t, \varepsilon)>0$ such that for every open set $D \subset X$ and all $\omega \in \Omega$,

$$
\mathbf{E}_{x}\left(\chi_{D_{h}}\left(X_{t}\right) \chi_{\left\{\tau_{x}^{D}<t\right\}}\right) \leqslant \varepsilon
$$

for almost every $x \in D_{h}$.

(iii) For every random operator $\left\{H_{\omega}\right\}$ and for all $t, \varepsilon>0$, there exists an $r=r(t, \varepsilon, H)>0$ such that for every open set $D \subset X$ and all $\omega \in \Omega$, we have

$$
0 \leqslant k_{\omega}(t, x, y)-k_{\omega}^{D}(t, x, y) \leqslant \varepsilon
$$

for almost all $x, y \in D_{r}$.

Proof. (i) $\Rightarrow$ (ii). By the Feynman-Kac formula for the unperturbed $\Delta_{\omega}$-operator, we have

$$
\mathbf{E}_{x}\left(\chi_{D_{h}}\left(X_{t}\right) \chi_{\left\{\tau_{x}^{D}<t\right\}}\right)=\int\left[k_{\Delta_{\omega}}(t, x, y)-k_{\Delta_{\omega}^{D}}(t, x, y)\right] \chi_{D_{h}}(y) d y
$$

which can be bounded, using the Hölder inequality and domain monotonicity, by

$$
\begin{gathered}
\left.\underset{y \in D_{h}}{\operatorname{ess} \sup _{0}} \mid k_{\Delta_{\omega}}(t, x, y)-k_{\Delta_{\omega}^{D}}(t, x, y)\right)\left.\right|^{1 / 2} \int\left(k_{\Delta_{\omega}}(t, x, y)-k_{\Delta_{\omega}^{D}}(t, x, y)\right)^{1 / 2} \chi_{D_{h}}(y) d y \\
\leqslant \underset{y \in D_{h}}{\operatorname{ess} \sup _{\Delta_{\omega}}}\left|k_{\Delta_{\omega}}(t, x, y)-k_{\Delta_{\omega}^{D}}(t, x, y)\right|^{1 / 2} \int k_{\Delta_{\omega}}(t, x, y)^{1 / 2} \chi_{D_{h}}(y) d y .
\end{gathered}
$$

The first term can be seen to be small by (i) for almost every $x \in D_{h}$, and the second term is bounded by $B_{t, 1 / 2}$, due to Proposition 5.1(ii).

(ii) $\Rightarrow$ (iii). We have to show that

$$
\chi_{D_{h}}\left(\exp \left(-t H_{\omega}\right)-\exp \left(-t H_{\omega}^{D}\right)\right) \chi_{D_{h}}: L^{1}\left(D_{h}, \operatorname{vol}_{\omega}\right) \rightarrow L^{\infty}\left(D_{h}, \operatorname{vol}_{\omega}\right)
$$

is arbitrarily small for $h$ large enough (independently of $\omega$ and $D$ ). Let $R:=\exp \left(-\frac{1}{2} t H_{\omega}\right)-\exp \left(-\frac{1}{2} t H_{\omega}^{D}\right)$. Note that

$$
\left\|e^{-t H_{\omega}^{D} / 2}\right\|_{1 \rightarrow 2} \leqslant \sqrt{B_{t / 2,2}}
$$

by Corollary 5.2. Thus, since

$$
\chi_{D_{h}}\left(e^{-t H_{\omega}}-e^{-t H_{\omega}^{D}}\right) \chi_{D_{h}}=\chi_{D_{h}} e^{-t H_{\omega} / 2} R \chi_{D_{h}}+\chi_{D_{h}} R e^{-t H_{\omega}^{D} / 2} \chi_{D_{h}}
$$

and by duality

$$
\left\|R \chi_{D_{h}}\right\|_{1 \rightarrow 2}=\left\|\chi_{D_{h}} R\right\|_{2 \rightarrow \infty}
$$


it suffices to show that

$$
\chi_{D_{h}}\left(\exp \left(-t H_{\omega}\right)-\exp \left(-t H_{\omega}^{D}\right)\right) \chi_{D_{h}}: L^{2}\left(D_{h}, \operatorname{vol}_{\omega}\right) \rightarrow L^{\infty}\left(D_{h}, \operatorname{vol}_{\omega}\right)
$$

is arbitrarily small for $h$ large enough (independently of $\omega$ and $D$ ). Using the Feynman-Kac formula for the perturbed operator, we obtain

$$
\begin{aligned}
(\exp ( & \left.\left.-t H_{\omega}\right)-\exp \left(-t H_{\omega}^{D}\right)\right) f(x) \\
& =\mathbf{E}_{x}\left(\exp \left(-\int_{0}^{t} V_{\omega}\left(X_{s}\right) d s\right) \chi_{D_{h}}\left(X_{t}\right) f\left(X_{t}\right) \chi_{\left\{\tau_{D}^{x}<t\right\}}\right) \\
& \leqslant \mathbf{E}_{x}\left(f\left(X_{t}\right)^{2}\right)^{1 / 2} \mathbf{E}_{x}\left(\chi_{D_{h}}\left(X_{t}\right) \chi_{\left\{\tau_{D}^{x}<t\right\}}\right)^{1 / 2} \\
& \leqslant\left(\int_{X} k_{\omega}(t, x, y)|f(y)|^{2} d y\right)^{1 / 2} \mathbf{E}_{x}\left(\chi_{D_{h}}\left(X_{t}\right) \chi_{\left\{\tau_{D}^{x}<t\right\}}\right)^{1 / 2} \\
& \leqslant C_{t}^{1 / 2}\|f\|_{2} \mathbf{E}_{x}\left(\chi_{D_{h}}\left(X_{t}\right) \chi_{\left\{\tau_{D}^{x}<t\right\}}\right)^{1 / 2} .
\end{aligned}
$$

The proof is finished by invoking (ii).

(iii) $\Rightarrow$ (i). This is immediate on choosing $V_{\omega} \equiv 0$.

The following lemma is an adaptation of Proposition 1.1 in [35, Chapter 6]. It is useful in our proof of 'not feeling the boundary'.

LEMMA 6.2 (Maximum principle for heat equation with non-negative potential). Let $D \subset X$ be open with compact closure, $V \geqslant 0$, and

$$
u \in C([0, T) \times \bar{D}) \cap C^{2}((0, T) \times D)
$$

be a solution of the heat equation

$$
\frac{\partial}{\partial t} u+(\Delta+V) u=0 \quad \text { on }(0, T) \times D
$$

with non-negative supremum $s=\sup \{u(t, x) \mid(t, x) \in[0, T) \times \bar{D}\}$. Then,

$$
s=\max \left\{\max _{x \in \bar{D}} u(0, x), \sup _{[0, T) \times \partial D} u(t, x)\right\} .
$$

Proof. It suffices to prove that, for any $c \geqslant 0$, the assumption

$$
u<c \text { on }(\{0\} \times \bar{D}) \cup([0, T) \times \partial D)
$$

implies that $u \leqslant c$ on $[0, T) \times D$. Towards this aim we introduce the auxiliary function $u_{\delta}(t, x)=u(t, x)-\delta t$, for $\delta>0$, and show that (15) implies that $u_{\delta}\left(t_{0}, x_{0}\right)<c$ on $[0, T) \times D$.

Assume that the conclusion is wrong. Then there exists $\left(t_{0}, x_{0}\right) \in(0, T) \times D$ such that $u_{\delta}\left(t_{0}, x_{0}\right) \geqslant c$. By continuity, the function $f(t):=\max _{x \in \bar{D}} u_{\delta}(t, x)$ is well defined and $t_{1}:=\min _{t \geqslant 0}\{t \mid f(t)=c\}$ exists. By (15), we have $0<t_{1} \leqslant t_{0}$, and there exists an $x_{1} \in D$ such that $u_{\delta}\left(t_{1}, x_{1}\right)=c$.

On the one hand, we have $\left(\partial u_{\delta} / \partial t\right)\left(t_{1}, x_{1}\right) \geqslant 0$ and, on the other, since $u_{\delta}\left(t_{1}, \cdot\right)$ has a global maximum at $x_{1},\left(\Delta_{x} u_{\delta}\right)\left(t_{1}, x_{1}\right) \geqslant 0$. Evaluating at $\left(t_{1}, x_{1}\right)$ yields the 
desired contradiction:

$$
0 \leqslant \frac{\partial u_{\delta}}{\partial t}=\frac{\partial u}{\partial t}-\delta=-\Delta u-V u-\delta \leqslant-\delta<0 .
$$

We now prove the principle of not feeling the boundary for the free Laplacian using an idea of H. Weyl (cf. [11, Lemma 3.5] for a Euclidean version).

Proposition 6.3. For any fixed $t, \varepsilon>0$, there exists an $h=h(t, \varepsilon)>0$ such that, for every open set $D \subset X$ and all $\omega \in \Omega$,

$$
0 \leqslant k_{\Delta_{\omega}}(t, x, y)-k_{\Delta_{\omega}^{D}}(t, x, y) \leqslant \varepsilon,
$$

for all $x \in D$ and $y \in D_{h}$.

Proof. We prove that the proposition is true for any $h>0$ satisfying

$$
C_{t} \exp \left(-\alpha_{t} C_{g}^{-2}\left(\frac{1}{2} h\right)^{2}\right) \leqslant \varepsilon .
$$

Let $\omega \in \Omega$ be fixed, and $f_{\delta} \in C_{0}^{\infty}\left(B_{\delta}(y)\right)$, with $0<\delta<\frac{1}{2} h$, be a non-negative approximation of the $\delta_{y}$-distribution at $y \in D_{h}$. Here, $B_{\delta}(y)$ denotes the open $d_{\omega}$-ball around $y$ with radius $\delta$. Denote by $k(t, x, y)=k_{\Delta_{w}}(t, x, y)$ the heat kernel of the semigroup $e^{-t \Delta_{\omega}}$ and set

$$
u_{1}(t, x):=\int_{X} k(t, x, z) f_{\delta}(z) d \operatorname{vol}_{\omega}(z)=\int_{D} k(t, x, z) f_{\delta}(z) d \operatorname{vol}_{\omega}(z) .
$$

Moreover, let $k^{D}(t, x, y)=k_{\Delta_{\omega}^{D}}(t, x, y)$ be the heat kernel of the semigroup $e^{-t \Delta_{\omega}^{D}}$ on $D$ with Dirichlet data on the boundary $\partial D$, and set

$$
u_{2}(t, x):=\int_{D} k^{D}(t, x, z) f_{\delta}(z) d \operatorname{vol}_{\omega}(z) .
$$

The difference $u_{1}(t, x)-u_{2}(t, x)$ solves the differential equation

$$
\left(\frac{\partial}{\partial t}+\Delta_{\omega}\right) u=0
$$

and satisfies the initial condition $u_{1}(0, x)-u_{2}(0, x)=f_{\delta}(x)-f_{\delta}(x)=0$ for all $x \in D$. Now, by domain monotonicity we know $k(t, x, z)-k^{D}(t, x, z) \geqslant 0$, and thus

$$
u_{1}(t, x)-u_{2}(t, x)=\int_{D}\left(k(t, x, z)-k^{D}(t, x, z)\right) f_{\delta}(z) d \operatorname{vol}_{\omega}(z) \geqslant 0
$$

for all $t>0$ and $x \in D$. The application of the maximum principle yields

$$
u_{1}(t, x)-u_{2}(t, x) \leqslant \max _{(0, t] \times \partial D}\left\{u_{1}(s, w)-u_{2}(s, w)\right\} .
$$

The expression on the right-hand side can be further estimated as:

$$
\begin{aligned}
u_{1}(s, w)-u_{2}(s, w) & \leqslant \int_{D} k(s, w, z) f_{\delta}(z) d \operatorname{vol}_{\omega}(z) \\
& =\int_{D_{h / 2}} k(s, w, z) f_{\delta}(z) d \operatorname{vol}_{\omega}(z) .
\end{aligned}
$$


Since $w \in \partial D$ and $z \in D_{h / 2}$, we conclude with (13) in Proposition 5.1:

$$
\int_{D_{h / 2}} k(s, w, z) f_{\delta}(z) d \operatorname{vol}_{\omega}(z) \leqslant C_{t} \exp \left(-\alpha_{t} C_{g}^{-2}\left(\frac{1}{2} h\right)^{2}\right) \leqslant \varepsilon .
$$

Taking the limit $\delta \rightarrow 0$, proves the proposition.

\section{Construction of the IDS by an exhaustion procedure}

Using the strategy of [28], we show that the IDS, defined in (2), coincides with the limit of an exhaustion procedure, for almost all $\omega \in \Omega$. This proves the selfaveraging property of the IDS stated in Theorem 4.

We first introduce the notion of an admissible sequence of subsets of $X$. As explained in $[\mathbf{1}, \S 3]$, let $\mathcal{F} \subset X$ be a polyhedral fundamental domain of the group $\Gamma$. Any finite subset $I \subset \Gamma$ defines a corresponding set

$$
\phi(I):=\operatorname{int}\left(\bigcup_{\gamma \in I} \gamma \overline{\mathcal{F}}\right) \subset X
$$

Now, admissible sequences are defined via tempered Følner sequences.

Definition 7.1. (a) A sequence $\left\{I_{j}\right\}_{j}$ of finite subsets in $\Gamma$ is called a Følner sequence if

$$
\lim _{j \rightarrow \infty} \frac{\left|I_{j} \Delta I_{j} \gamma\right|}{\left|I_{j}\right|}=0 \quad \text { for all } \gamma \in \Gamma .
$$

(b) A Følner sequence $\left\{I_{j}\right\}_{j}$ is called a tempered Følner sequence if it is monotonically increasing and satisfies

$$
\sup _{j \in \mathbb{N}} \frac{\left|I_{j+1} I_{j}^{-1}\right|}{\left|I_{j+1}\right|}<\infty .
$$

(c) A sequence $\left\{D^{j}\right\}_{j}$ of subsets of $X$ is called admissible if there exists a tempered Følner sequence $\left\{I_{j}\right\}_{j}$ in $\Gamma$ with $D^{j}=\phi\left(I_{j}\right)$, for $j \in \mathbb{N}$.

By Lemma 2.4 in [28], an admissible sequence satisfies the isoperimetric property

$$
\lim _{j \rightarrow \infty} \frac{\operatorname{vol}_{0}\left(\partial_{d} D^{j}\right)}{\operatorname{vol}_{0}\left(D^{j}\right)}=0, \quad \text { for all } d>0 .
$$

Existence of a Følner sequence is a geometrical description of amenability of the group $\Gamma$. The notion of 'tempered Følner sequence' is due to A. Shulman [31] and used by Lindenstrauss in the proof of the following pointwise ergodic theorem [25].

THEOREM 7. Let $G$ be a finitely generated discrete amenable group.

(a) The group $G$ admits monotonically increasing Følner sequences.

(b) Every monotonically increasing Følner sequence has a tempered subsequence.

(c) Assume that $G$ acts ergodically on $\Omega$ by measure-preserving transformations $\left\{T_{\gamma}\right\}_{\gamma}$. Let $\left\{I_{j}\right\}_{j}$ be a tempered Følner sequence. Then we have, for 
every $f \in L^{1}(\Omega)$,

$$
\lim _{j \rightarrow \infty} \frac{1}{\left|I_{j}\right|} \sum_{\gamma \in I_{j}^{-1}} f\left(T_{\gamma} \omega\right)=\mathbb{E}(f)
$$

in almost-sure and $L^{1}$-topology.

The results of the last two sections are used to prove the following heat kernel lemma.

Lemma 7.2. Let $\left\{D^{j}\right\}$, for $j \in \mathbb{N}$, be an admissible sequence and let $\left\{H_{\omega}\right\}_{\omega}$ be a random operator. Then the following hold:

(a) $\sup _{\omega \in \Omega} \operatorname{vol}_{\omega}\left(D^{j}\right)^{-1}\left|\operatorname{tr}\left(\chi_{D^{j}} \exp \left(-t H_{\omega}\right)\right)-\operatorname{tr}\left(\exp \left(-t H_{\omega}^{j}\right)\right)\right| \rightarrow 0$, as $j \rightarrow \infty$;

(b) there exists a constant $C>0$ with $\operatorname{tr}\left(\chi_{\mathcal{F}} e^{-t H_{\omega}}\right) \leqslant C$ for all $\omega \in \Omega$;

(c) the map $\omega \mapsto \operatorname{tr}\left(\chi_{\mathcal{F}} e^{-t H_{\omega}}\right)$ is measurable.

Proof. (a) Since $\exp (-t H)=\exp \left(-\frac{1}{2} t H\right) \exp \left(\frac{1}{2} t H\right)$ for arbitrary $H \geqslant 0$ and by using standard calculations for integral kernels, we have

$$
\operatorname{tr}\left(\chi_{D^{j}} e^{-t H_{\omega}}\right)=\int_{D^{j}} \int_{D^{j}} k_{\omega}\left(\frac{1}{2} t, x, y\right)^{2} d \operatorname{vol}_{\omega}(x) d \operatorname{vol}_{\omega}(y)
$$

and

$$
\operatorname{tr}\left(e^{-t H_{\omega}^{j}}\right)=\int_{D^{j}} \int_{D^{j}} k_{H_{\omega}^{j}}\left(\frac{1}{2} t, x, y\right)^{2} d \operatorname{vol}_{\omega}(x) d \operatorname{vol}_{\omega}(y) .
$$

We express the difference of (19) and (20) using the fact that

$$
k_{\omega}^{2}-k_{H_{\omega}^{j}}^{2}=\left[k_{\omega}-k_{H_{\omega}^{j}}\right]\left[k_{\omega}+k_{H_{\omega}^{j}}\right]
$$

and use the following decomposition of the integration domain:

$$
\begin{gathered}
\int_{D^{j}} \int_{D^{j}}\left[k_{\omega}\left(\frac{1}{2} t, x, y\right)-k_{H_{\omega}^{j}}\left(\frac{1}{2} t, x, y\right)\right]\left[k_{\omega}\left(\frac{1}{2} t, x, y\right)+k_{H_{\omega}^{j}}\left(\frac{1}{2} t, x, y\right)\right] d \operatorname{vol}_{\omega}(x, y) \\
=\int_{\partial_{h} D^{j}} \int_{\partial_{h} D^{j}} \ldots+\int_{D_{h}^{j}} \int_{\partial_{h} D^{j}} \ldots+\int_{\partial_{h} D^{j}} \int_{D_{h}^{j}} \ldots+\int_{D_{h}^{j}} \int_{D_{h}^{j}} \ldots
\end{gathered}
$$

Each of the first three terms can be bounded by $2 C_{t / 2} B_{t / 2} \operatorname{vol}_{\omega}\left(\partial_{h} D^{j}\right)$ by inferring the following consequences of $\S 5$ :

$$
0 \leqslant k_{H_{\omega}^{j}}\left(\frac{1}{2} t, x, y\right) \leqslant k_{\omega}\left(\frac{1}{2} t, x, y\right) \leqslant C_{t / 2} \quad \text { and } \quad \int k_{\omega}\left(\frac{1}{2} t, x, y\right) d \operatorname{vol}_{\omega}(y) \leqslant B_{t / 2}
$$

for almost every $x, y \in X$. As for the last term, we fix $\varepsilon>0$ and choose $h=h\left(\frac{1}{2} t, \varepsilon\right)$ according to Theorem 6 and obtain the bound $2 \varepsilon B_{t / 2} \operatorname{vol}_{\omega}\left(D^{j}\right)$. By $(5)$, we conclude that the sequence $D^{j}$ satisfies the isoperimetric property

$$
\lim _{j \rightarrow \infty} \frac{\operatorname{vol}_{\omega}\left(\partial_{d} D^{j}\right)}{\operatorname{vol}_{\omega}\left(D^{j}\right)}=0, \quad \text { for all } d>0,
$$

for the metric $g_{\omega}$, as well. This shows part (a).

Now, (b) follows from (5), Proposition 5.1(ii) and the analog of (19) for $\chi_{\mathcal{F}}$, while (c) follows from measurability of $\omega \mapsto e^{-t H_{\omega}}$, after choosing a suitable orthonormal basis according to Appendix A of [21]. 
Finally, we present the proof of our main result.

Proof of Theorem 4. A criterion of Pastur and Šubin [26, 33] establishes the convergence of the normalized eigenvalue counting functions $N_{\omega}^{j}$ to a selfaveraging limit, if their Laplace transforms $\mathcal{L}_{\omega}^{j}(\cdot)$ converge. To apply this criterion (cf. [28, 21], as well) we note that the random operator $\left\{H_{\omega}\right\}_{\omega}$ is non-negative and, by Lemma $7.2(\mathrm{~b})$, the $\mathcal{L}_{\omega}^{j}(t)$ are bounded by a constant depending only on $t$. So it remains to show that

$$
\lim _{j \rightarrow \infty} \mathcal{L}_{\omega}^{j}(t):=\lim _{j \rightarrow \infty} \int_{\mathbb{R}} e^{-t \lambda} d N_{\omega}^{j}(\lambda)=\int_{\mathbb{R}} e^{-t \lambda} d N_{H}(\lambda)
$$

for all $t>0$, in $L^{1}$ and $\mathbb{P}$-almost-sure sense. This is done by applying Lindenstrauss' ergodic theorem (Theorem 7 ). We introduce the equivalence relation $a_{j} \sim_{j \rightarrow \infty} b_{j}$ for two arbitrary sequences $a_{j}(\omega)$ and $b_{j}(\omega)$, with $j \in \mathbb{N}$, satisfying $a_{j}-b_{j} \rightarrow 0$, as $j \rightarrow \infty$, in $L^{1}$ and $\mathbb{P}$-almost surely. By definition we have

$$
\mathcal{L}_{\omega}^{j}(t)=\operatorname{vol}_{\omega}\left(D^{j}\right)^{-1} \operatorname{tr}\left(e^{-t H_{\omega}^{j}}\right) .
$$

Using the previous lemma, equivariance and Theorem 7, we derive

$$
\begin{aligned}
\left|I_{j}\right|^{-1} \operatorname{tr}\left(e^{-t H_{\omega}^{j}}\right) & \sim_{j \rightarrow \infty}\left|I_{j}\right|^{-1} \operatorname{tr}\left(\chi_{D^{j}} e^{-t H_{\omega}}\right)=\left|I_{j}\right|^{-1} \sum_{\gamma \in I_{j}} \operatorname{tr}\left(\chi_{\gamma \mathcal{F}} e^{-t H_{\omega}}\right) \\
& =\left|I_{j}\right|^{-1} \sum_{\gamma \in I_{j}^{-1}} \operatorname{tr}\left(\chi_{\mathcal{F}} e^{-t H_{\gamma \omega}}\right) \sim_{j \rightarrow \infty} \mathbb{E}\left(\operatorname{tr}\left(\chi_{\mathcal{F}} e^{-t H_{\bullet}}\right)\right) .
\end{aligned}
$$

Similarly, we infer that

$$
\begin{aligned}
\left|I_{j}\right|^{-1} \operatorname{vol}_{\omega}\left(D^{j}\right) & =\left|I_{j}\right|^{-1} \sum_{\gamma \in I_{j}} \operatorname{vol}_{\omega}(\gamma \mathcal{F}) \\
& =\left|I_{j}\right|^{-1} \sum_{\gamma \in I_{j}^{-1}} \operatorname{vol}_{\gamma \omega}(\mathcal{F}) \sim_{j \rightarrow \infty} \mathbb{E}\left\{\operatorname{vol}_{\bullet}(\mathcal{F})\right\} .
\end{aligned}
$$

Putting this together, and noting that, by (5),

$$
C_{g}^{-n / 2} \operatorname{vol}_{0}(\mathcal{F}) \leqslant|I|^{-1} \operatorname{vol}_{\omega}(\phi(I)) \leqslant C_{g}^{n / 2} \operatorname{vol}_{0}(\mathcal{F}),
$$

for all finite sets $I \subset \Gamma$, we obtain

$$
\mathcal{L}_{\omega}^{j}(t)=\operatorname{vol}_{\omega}\left(D^{j}\right)^{-1} \operatorname{tr}\left(e^{-t H_{\omega}^{j}}\right)=\frac{\left|I_{j}\right|^{-1} \operatorname{tr}\left(e^{-t H_{\omega}^{j}}\right)}{\left|I_{j}\right|^{-1} \operatorname{vol}_{\omega}\left(D^{j}\right)} \sim_{j \rightarrow \infty} \frac{\mathbb{E}\left\{\operatorname{tr}\left(\chi_{\mathcal{F}} e^{-t H_{\bullet}}\right)\right\}}{\mathbb{E}\left\{\operatorname{vol}_{\bullet}(\mathcal{F})\right\}} .
$$

By (2),

$$
\frac{\mathbb{E}\left\{\operatorname{tr}\left(\chi_{\mathcal{F}} e^{-t H_{\bullet}}\right)\right\}}{\mathbb{E}\left\{\operatorname{vol}_{\bullet}(\mathcal{F})\right\}}=\int_{\mathbb{R}} e^{-t \lambda} d N_{H}(\lambda) .
$$

This completes the proof.

Acknowledgements. It is a pleasure to thank B. Franke, D. Hundertmark, L. Karp, W. Kirsch, O. Post and P. Stollmann for stimulating discussions. This work was supported in part by the DFG through SFB 237 'Unordnung und große Fluktuationen' and the Schwerpunktprogramm 'Interagierende stochastische 
Systeme von hoher Komplexität'. I. Veselić thanks B. Simon for hospitality at CalTech.

\section{References}

1. T. Adachi and T. Sunada, 'Density of states in spectral geometry', Comment. Math. Helv. 68 (1993) 480-493.

2. M. Aizenman and S. Molchanov, 'Localization at large disorder and at extreme energies: an elementary derivation', Comm. Math. Phys. 157 (1993) 245-278.

3. P. W. Anderson, 'Absence of diffusion in certain random lattices', Phys. Rev. 109 (1958) 1492.

4. H. Bauer, Maß- und Integrationstheorie, 2nd edn (de Gruyter, Berlin, 1992).

5. R. CARmona and J. LaCroIX, Spectral theory of random Schrödinger operators (Birkhäuser, Boston, 1990).

6. I. Chavel, Eigenvalues in Riemannian geometry (Academic Press, New York, 1984).

7. I. Chavel, Riemannian geometry - a modern introduction (Cambridge University Press, 1993).

8. H. L. Cycon, R. G. Froese, W. Kirsch and B. Simon, Schrödinger operators with application to quantum mechanics and global geometry, Text and Monographs in Physics (Springer, Berlin, 1987).

9. E. B. Davies, Heat kernels and spectral theory (Cambridge University Press, 1989).

10. E. B. DAvies, 'Spectral properties of compact manifolds and changes of metric', Amer. J. Math. 112 (1990) 15-39.

11. J. Dodziuk, 'Eigenvalues of the Laplacian and the heat equation', Amer. Math. Monthly 88 (1981) 686-695.

12. J. DodzIUK, 'Maximum principle for parabolic inequalities and the heat flow on open manifolds', Indiana Univ. Math. J. 32 (1983) 704-716.

13. K. D. Elworthy, Stochastic differential equations on manifolds, London Mathematical Society Lecture Note Series 70 (Cambridge University Press, 1982).

14. J. Fröhlich and T. Spencer, 'Absence of diffusion in the Anderson tight binding model for large disorder or low energy', Commun. Math. Phys. 88 (1983) 151-184.

15. I. Ya. Goldsheid, S. A. MolČanov and L. A. Pastur, 'A pure point spectrum of the stochastic one-dimensional Schrödinger operator', Functional Anal. Appl. 11 (1977) 1-10.

16. T. Kato, Perturbation theory for linear operators (Springer, Berlin, 1980).

17. W. Kirsch, 'Random Schrödinger operators', Schrödinger operators (ed. H. Holden and A. Jensen), Lecture Notes in Physics 345 (Springer, Berlin, 1989).

18. W. Kirsch and F. Martinelli, 'On the ergodic properties of the spectrum of general random operators', J. Reine Angew. Math. 334 (1982) 141-156.

19. F. Kleespies and P. Stollmann, 'Lifshitz asymptotics and localization for random quantum waveguides', Rev. Math. Phys. 12 (2000) 1345-1365.

20. D. Lenz, N. Peyerimhoff, O. Post and I. Veselić, 'Continuity properties of the integrated density of states on manifolds', in preparation.

21. D. Lenz, N. Peyerimhoff and I. Veselić, 'Von Neumann algebras, groupoids and the integrated density of states', Preprint, arXiv:math-ph/0203026, 2002.

22. D. Lenz, N. Peyerimhoff and I. Veselić, 'Random Schrödinger operators on manifolds', Markov Process. Related Fields to appear, arXiv:math-ph/0212057.

23. P. LI and S. T. YAU, 'On the parabolic kernel of the Schrödinger operator', Acta Math. 156 (1986) $153-201$.

24. I. Lifshitz, 'The energy spectrum of disordered systems', Adv. Phys. 13 (1964) 483-536.

25. E. Lindenstrauss, 'Pointwise theorems for amenable groups', Invent. Math. 146 (2001) $259-295$.

26. L. A. PASTuR, 'Selfaverageability of the number of states of the Schrödinger equation with a random potential', Mat. Fiz. i Funkcional. Anal. (Vyp. 2) 238 (1971) 111-116.

27. L. A. Pastur and A. L. Figotin, Spectra of random and almost-periodic operators (Springer, Berlin, 1992).

28. N. Peyerimhoff and I. Veselić, 'Integrated density of states for ergodic random Schrödinger operators on manifolds', Geom. Dedicata 91 (2002) 117-135.

29. M. Reed and B. Simon, Methods of modern mathematical physics II, Fourier analysis, self-adjointness (Academic Press, San Diego, 1975).

30. M. Reed and B. Simon, Methods of modern mathematical physics I, functional analysis (Academic Press, San Diego, 1980).

31. A. Shulman, 'Maximal ergodic theorems on groups', Dep. Lit. NIINTI (1988) 2184. 
32. P. Stollmann, Caught by disorder: a course on bound states in random media, Progress in Mathematical Physics 20 (Birkhäuser, Boston, 2001).

33. M. A. Šubin, 'Spectral theory and the index of elliptic operators with almost-periodic coefficients', Uspekhi Mat. Nauk 34 (1979) 95-135 (Russian), Russian Math. Surveys 34 (1979) 109-157 (English).

34. M. A. Subin, 'Density of states of self adjoint operators with almost periodic coefficients', Amer. Math. Soc. Transl. 118 (1982) 307-339.

35. M. E. TAYlor, Partial differential equations I, basic theory (Springer, New York, 1996).

36. F. Wegner, 'Bounds on the DOS in disordered systems', Z. Phys. B 44 (1981) 9-15.

Daniel Lenz

Fakultät für Mathematik

TU Chemnitz

09107 Chemnitz

Germany

dlenz@mathematik.tu-chemnitz.de

www.tu-chemnitz.de/mathematik/analysis/dlenz

Norbert Peyerimhoff

Fakultät für Mathematik

Ruhr-Universität Bochum

44780 Bochum

Germany

peyerim@math.ruhr-uni-bochum.de

www.ruhr-uni-bochum.de/mathematik10/Norbert.html

Ivan Veselić

Forschungsstipendiat der Deutschen Forschungsgemeinschaft

Current address:

Department of Mathematics

California Institute of Technology

Pasadena

CA 91125

USA

ivan.veselic@ruhr-uni-bochum.de

http://homepage.ruhr-uni-bochum.de/Ivan.Veselic/ 